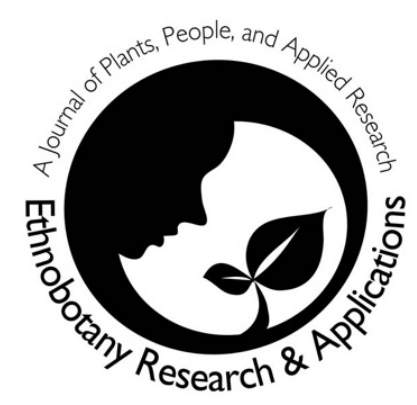

\title{
Importance sociale de Bambusa vulgaris Schrad ex. J.C. Wendl. (Poaceae) dans la Sous-préfecture d'Azaguié, Sud-Est de la Côte d'Ivoire
}

\author{
Dobo Pierre Valence Dje Bi, Kouao Jean Koffi et Constant \\ Yves Adou Yao
}

\section{Research}

\begin{abstract}
Résumé
Contexte: Bambusa vulgaris Schrad ex. J.C. Wendl. (Poaceae) encore appelé le bambou de Chine ou «" l'or vert " est une espèce polyvalente largement utilisée dans le monde en général et particulièrement en Afrique par communautés locales. La présente étude porte sur les différents usages et valeurs d'usage de Bambusa vulgaris dans la souspréfecture d'Azaguié au Sud-Est de la Côte d'Ivoire, où l'espèce est largement répandue.
\end{abstract}

Méthodes: Au total, 258 personnes ont été interrogées dans la zone d'étude à l'aide d'un questionnaire semi-structuré. Les valeurs d'usage de la plante en fonction des différents groupes ethniques, des localités, de l'âge des populations de la zone d'étude ont été déterminées à travers les calculs de fréquences et de moyennes (Test de Kruskal-Wallis). De plus les valeurs d'usage des organes de la plante ont été évaluées en détail.

Résultats: Toutes les personnes interrogées dans la zone d'étude connaissaient au moins une utilisation de B. vulgaris. En somme, 38 usages ont été mentionnés regroupés en dix (10) catégories. Les principaux domaines d'utilisations identifiées ont été la construction $(84,38 \%)$, le bois énergie $(70,51 \%)$, l'agriculture $(61,27 \%)$. Le chaume est l'organe le plus impliqué dans ces types d'utilisation. Les valeurs consensuelles du groupe ethnique pour les organs de $B$. vulgaris ont montré que le chaume est le plus utilisé. Les connaissances concernant l'utilisation de $B$. vulgaris sont réparties de manière homogène entre les groupes ethniques et les localités. B. vulgaris est bien connu et utilisé de différentes manières par les populations locales de la zone d'étude. Les connaissances locales sur l'espèce sont diversifiées et le plus souvent influencées par l'âge au niveau des vieillards. II n'y a pas de différences significatives dans les valeurs d'usage et les modes d'utilisation de l'espèce aussi bien entre les groupes ethniques qu'entre les localités.

Conclusion: B. vulgaris est diversement utilisée dans la Sous-préfecture d'Azaguié.

Mots-clés: Bambusa vulgaris - usages - valeurs d'usage - sous-préfecture Azaguié - Côte d'Ivoire.

\section{Correspondence}

Dobo Pierre Valence Dje $\mathrm{Bi}^{1}$, Kouao Jean Koffi ${ }^{2^{*}}$ et Constant Yves Adou Yao ${ }^{3}$

${ }^{1}$ Laboratoire de Botanique UFR Biosciences, Université Félix Houphouët-Boigny (UFHB), 22 BP 582 Abidjan 22, Côte d'Ivoire. Tel : (+225) 47589663 / (+225) 02776786

${ }^{2}$ Laboratoire de Botanique, UFR Sciences de la Nature, Université Nangui Abrogoua (UNA), 02 BP 801 Abidjan 02 Côte d'Ivoire

.${ }^{3}$ Centre Suisse de Recherches Scientifiques en Côte d'Ivoire.

*Corresponding Author: kouaojean@yahoo.fr

Ethnobotany Research \& Applications 19:10 (2020) 


\begin{abstract}
Background: Chinese bamboo or Bambusa vulgaris Schrad ex. J.C. Wendl. (Poaceae) or "green gold" is a versatile species widely used in the world and particularly in Africa of local communities. The present study deals with the different uses and use values of $B$. vulgaris in the sub-prefecture of Azaguie in the south-east of Côte d'Ivoire, where the species grows widely.
\end{abstract}

Methods: Consumption values according to the different ethnic groups, the locality, the age of the population, the remaining intervention zone (KruskalWallis test). In addition, the values of the organs of the plant have been evaluated in detail.

Results: Among the ethnic groups, 258 people were interviewed in the study area with the help of questionnaires. All respondents in the study area knew at least one use of $B$. vulgaris. In total, 38 uses were mentioned here in ten (10) categories. The main areas of use identified were construction $(84.38 \%)$, wood energy $(70.51 \%)$ and agriculture $(61.27 \%)$. The stubble was the organ (part) The consensual values of the ethnic group for the parts of $B$. vulgaris were shown to stubble. Knowledge about the use of $B$. vulgaris is evenly distributed between ethnic groups and localities. $B$. vulgaris is used by local populations of the study area. Local knowledge is diverse and often influenced by age. There were no significant differences between values and usage patterns.

Conclusion: B. vulgaris is excessively used in the sub-prefecture of Azaguié.

Keywords: Bambusa vulgaris - uses - use values sub-prefecture Azaguié - Ivory Coast

\section{Contexte}

Plusieurs populations à travers le monde dépendent des ressources végétales et emploient leurs connaissances accumulées dans le temps pour en tirer profit et les gérer de façon durable (Sofowora 2010). Avant la fin des années 1970, beaucoup d'attention était accordée à la recherche sur les forêts et le développement de la productivité commerciale du bois sans tenir compte de son rôle réel et du potentiel des multiples produits et services environnementaux offerts par les forêts (Perez \& Byron 1999). Dans un passé récent, il y a eu un regain d'intérêt pour les Produits Forestiers Non Ligneux (PFNL). En effet, pour les populations surtout rurales, les PFNL revêtent une importance capitale car ils contribuent aux soins de santé, à l'alimentation, au revenu monétaire et aux autres aspects de leur bien-être (Mahapatra et al. 2005). Les PFNL sont des biens d'origine biologique autres que le bois, provenant des forêts, d'autres terrains boisés ou provenant d'arbres hors forêts (FAO 2001). Ceux-ci sont importants d'autant plus que leur valorisation et leur gestion durable pourraient efficacement contribuer à l'atteinte des Objectifs de Développement Durable (ODD) qui visent, entre autres, à éliminer la pauvreté, la faim et à assurer la sécurité alimentaire dans le monde (Fandohan et al. 2015, Ingram \& Bongers 2009, Lescuyer et al. 2010). On estime que $80 \%$ de la population mondiale en développement dépend largement des PFNL pour leurs moyens de subsistance (Shackleton \& Shackleton 2004). Aujourd'hui, ils jouent de plus en plus un rôle socio-économique, écologique, culturel et même des rôles politiques dans de nombreuses économies nationales dans les pays tropicaux. Au nombre de ces PFNL figure le bambou qui est une espèce herbacée de la famille des Poaceae. II est dans le monde la plante à croissance la plus rapide (Desalegn \& Tadesse 2014). Le genre Bambusa pousse de façon native sur cinq continents et renferme plus de 100 espèces dans les régions tropicales et subtropicales mondiales (Kleinhenz \& Midmore 2001). La croissance rapide du bambou, sa large disponibilité, ses usages sociaux, écologiques et économiques lui confèrent son importance ainsi que sa popularité (Ogunjinmi et al. 2009). Les divers usages du bambou (plus de 1500 documentés), font de lui une espèce mondialement connue (Desalegn \& Tadesse 2014). Quelquefois désigné sous le nom de "or vert" (Singh 2008), le bambou est profondément ancré dans la culture et les traditions asiatiques. C'est le reflet d'une longue histoire de culture remontant à l'âge néolithique (Ruiz-Perez et al. 2001). Ce n'est que dans ces dernières décennies que les ressources du bambou de la République Populaire de Chine ont cessé d'être utilisées principalement à des fins de subsistance et de commerce intérieur à petite échelle, pour devenir une marchandise au centre d'une industrie de plusieurs milliards de dollars par an (FAO 2007, Mertens et al. 2008). De plus, les nouvelles technologies de traitement post-récolte ont permis une expansion majeure des produits à base de tiges creuses de bambou. Cela a considérablement augmenté la demande et la production des pousses comestibles de bambou utilisées pour la conception de bière industrielle (FAO 2007, Mertens et al. 2008). II existe plusieurs domaines d'usages du bambou variant de la médecine à la nutrition en passant par la construction (Salam 2008). Les tiges de $B$. vulgaris sont utilisées dans la construction pour faire des palissades et surtout de petits abris temporaires (Ohrnberger 1999), y compris le plancher, la toiture, les panneaux et les clôtures faits avec des tiges fendues (Louppe et al. 2008). La tige est également utilisée dans la confection de certaines parties des bateaux y compris les mâts et les gouvernails. Dans 
l'artisanat, la tige est aussi utilisée pour faire le mobilier, la vannerie, les flûtes, la canne à pêche, les manches des outils, les pieux, les armes, les arcs pour les filets de pêche, les pipes de fumeur, etc (Dransfield \& Widjaja 1995). En nutrition, les jeunes pousses de la plante, cuites ou marinées, sont comestibles et mangées partout en Asie (Bhatt et al. 2005, Karanja et al. 2015, Nirmala et al. 2011). Ses usages sont nombreux en médecine indigène car il est considéré dans beaucoup de traditions à travers l'Asie pour sa valeur médicinale. En effet, à Java, l'eau entreposée dans ses tiges est utilisée comme une cure de plusieurs maladies (Louppe et al. 2008). L'intérieur des pousses de Bambusa vulgaris est utilisé pour guérir la jaunisse (Burkill 1935). En Chine, le jus de pousses du bambou pressées possède une protéase dont l'activité aide à la digestion de protéine. La décoction des pousses mélangée à l'huile de palme est utilisée pour la parturition ou indiquée pour l'avortement (Puri 2003). $\mathrm{Au}$ Congo, ses feuilles sont utilisées dans le traitement de la rougeole. Au Nigeria, l'infusion de feuilles macérées est abortive et sert à combattre les maladies sexuellement transmises (Yakubu \& Bukoye 2005). Au Ghana, les feuilles fraîches sont connues pour avoir des propriétés antibiotiques, comme assainir des taches moisies sur les œufs de poulet infectés (Darko Obiri \& Oteng-Amoako 2007).

Depuis son introduction en Côte d'Ivoire dans les années 1930, l'usage du bambou n'a cessé de prendre de l'ampleur. Mise en culture dans le Sud, Bambusa vulgaris est de plus en plus utilisé par les populations locales (Dje bi et al. 2017). Les travaux de N'Guessan (2008) et Koulibaly et al. (2016) ont évoqué l'usage du bambou dans la pharmacopée ivoirienne. Quant à Zadou et al. (2011), lors de leur étude portant sur l'inventaire des espèces trouvées à l'intérieur et aux alentours de la Forêt des Marais Tanoe-Ehy (FMTE), l'ont identifié comme une espèce régulièrement utilisée comme bois de chauffage par les populations riveraines de la FMTE. Malheureusement, toutes ces études n'ont presqu'établi aucune base de données sur les usages du bambou en Côte d'Ivoire en général et dans la localité d'Azaguié en particulier. La localité d'Azaguié et ses environs constituent la principale source d'approvisionnement en bambou. En effet, des entretiens avec les responsables de la Direction des Exploitations et des Industries Forestières (DEIF-Abidjan), ont confirmé que cette zone est la première en Côte d'Ivoire dans la production et l'exploitation du bambou. La présente étude a pour objectif de décrire les différents usages faits du bambou et évaluer son importance culturelle pour les différents groupes socioculturels observés dans une de ses zones majeures d'occurrence (Azaguié) en Côte d'Ivoire. Admettons que les usages et le degré de connaissance des plantes dépendent de la culture, des us et coutumes des peuples, de l'âge et du genre (Camou-Guerrero et al. 2008, Gouwakinnou et al. 2011). Spécifiquement, l'étude a permis (1) de catégoriser les usages du bambou dans la Sous-préfecture d'Azaguié, (2) d'analyser les différentes valeurs d'usages du bambou et (3) comparer les connaissances sur Bambusa vulgaris entre groupes socioculturels, catégories d'âges et genres.

\section{Matériel et Méthodes \\ Zone d'étude}

La présente étude a été conduite dans la Souspréfecture d'Azaguié, située à $40 \mathrm{~km}$ au Nord-Est de la ville d'Abidjan (Figure 1). Sur les sept villages que compte la Sous-préfecture d'Azaguié, quatre villages (Abbey-Beguini, Azaguié-Ahoua, Azaguié-Gare et M'Bromé) ont été les plus cités comme sources d'approvisionnement. II est à signaler que la ville d'Azaguié est la réunion deux villages (AzaguiéAhoua, Azaguié-Gare). De plus, ces localités ont été retenues dans le cadre de cette étude en raison du nombre élevé d'exploitants et d'usages qu'on y trouve. La Sous-préfecture d'Azaguié a une population estimée à plus de 30000 habitants (RGPH 2014). Elle est composée d'autochtones Abbey et d'allochtones originaires de diverses régions de la Côte d'Ivoire, notamment les Baoulés, les Malinkés, les Wê, etc. ainsi que des allogènes ressortissants des pays de la sous-région, notamment les Burkinabés, les Maliens, Guinéens. Comme activités socio-économiques à Azaguié, il existe beaucoup de plantations Agro-industrielles de fleurs, de banane dessert. Lors de la réalisation de ces plantations, les anciens occupants ont planté à l'époque, les bambous de chine afin de les délimiter et de servir de tuteur pour soutenir les régimes de banane. Outre cela, les populations d'Azaguié font plusieurs plantations d'hévéa, de papayes solos, de palmiers à huiles, de café, cacao, cola, ... Le manioc est la principale culture vivrière avec diverses variétés. On a aussi la pêche, le commerce, le transport, l'artisanat, mais surtout l'exploitation du bambou qui est une véritable source de revenu pour plusieurs personnes. La végétation originelle qui était une forêt dense sempervirente a disparu au fil du temps pour laisser place à de vastes espaces agricoles (Vroh et al. 2010). Les caractéristiques de la zone se prêtent bien aux conditions favorables (Larpkern et al. 2011) à la production du bambou : relief avec la présence de nombreux bas-fonds (N'Go et al. 2005), sols ferralitiques, remaniés sous forte pluviométrie. 


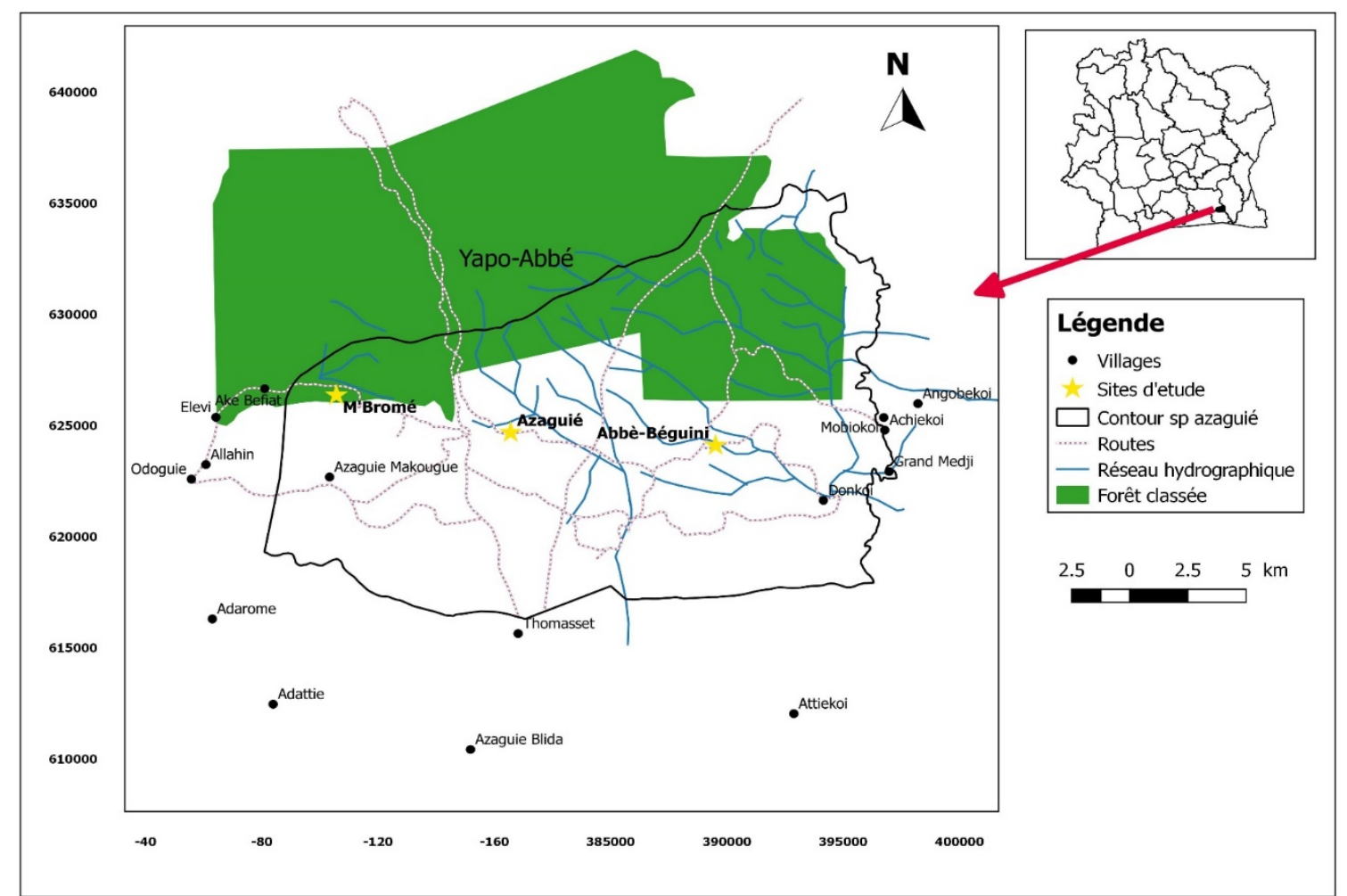

Figure 1. Localisation de la Sous-préfecture d'Azaguié dans la Région de l'Agnéby-Tiassa, Sud-Est de la Côte d'Ivoire. (Source: Dje Bi et al. 2017)

Figure 1. Localization of Azaguie sub-prefecture in Agneby-Tiassa Region, Southeast in Côte d'Ivoire. (Source: Dje Bi et al. 2017)

\section{Collecte de données \\ Echantillonnage}

La sélection des enquêtés s'est faite de façon aléatoire et simple. À cet effet, un sondage exploratoire sur un échantillon de 20 personnes prises au hasard dans chacun des quatre villages a permis de déterminer la proportion ( $p$ ) de ceux qui connaissent au moins un usage de $B$. vulgaris. La détermination du nombre d'enquêtés a alors été faite en utilisant la formule de Dagnelie (1998) :

$$
n=\left[U^{2}{ }_{1-\alpha / 2} \times p(1-p)\right] / d^{2}
$$

Où $n$ représente la taille de l'échantillon global; $\cup_{1-\alpha / 2}$ est la valeur de la variable normale pour une valeur de probabilité de $\alpha ; \bigcup_{1-\alpha / 2}=1,96$ si $\alpha=0,05 ; p$ est la proportion d'individus des villages; d est l'erreur marginale. L'enquête exploratoire a révélé qu'environ environ $49 \%, 63 \%, 56 \%$ et $58 \%$ des personnes interrogées connaissent et utilisent l'espèce dans les villages d'Azaguié-Ahoua, Azaguié-Gare, M'Bromé et Abbey-Beguini, respectivement. Ainsi, pour une marge d'erreur de $8 \%$, environ 57, 71, 64 et 66 personnes ont été sélectionnées et interviewées dans ces quatre villages en fonction de leur consentement libre à participer à l'enquête. Ces personnes ont été choisies au hasard dans ces localités.

\section{Enquêtes ethnobotaniques}

L'étude a été basée sur des enquêtes auprès des populations villageoises. Ces enquêtes ont été réalisées dans des localités d'Azaguié où le Bambou est à la fois exploité et utilisée. Au total, 258 personnes ont été interviewées dans les quatre villages sélectionnés de la Sous-préfecture d'Azaguié. La méthode d'enquête utilisée est celle des entretiens individuels semi-structurés et des observations directes (Tefera et al. 2013). Le questionnaire de base est structuré en deux parties. La première partie a renseigné le profil de l'enquêté (l'âge, le genre, le groupe socioculturel, ...) ainsi que sa profession ou sa principale activité. La seconde partie concerne, d'une part, les connaissances de l'enquêté sur les usages faits de l'espèce, de ses organes, d'autre part, l'importance culturelle de la plante et de ses différentes organes. Les catégories d'usages à cet effet sont adaptées de Fandohan et al. (2010) et de Tardio et Pardo-De-Santayana (2008): construction, alimentation, artisanat, pêche, élevage, agricuture, écologique, matériel (outil), médecine et socioculturel. L'évaluation de l'importance culturelle de la plante et de ses organes a été menée en utilisant une grille de score d'importance à quatre niveaux (Bélem 2009, Belem et al. 2008). En effet, dans un premier temps, il a été demandé à chaque enquêté d'attribuer un score 
d'importance à chaque catégorie d'usages pour la plante entière. Dans un second temps, le même exercice a été effectué pour chaque catégorie d'usages, mais cette fois par organe de plante. Les scores varient de 0 à 1,5. Le score 0 correspond à une catégorie d'usages non importante; 0,5 est attribué à une catégorie d'usages moyennement importante; 1 pour une catégorie d'usages importante; 1,5 est affecté au score maximal et correspond à une catégorie d'usages très importante.

Traitement et analyses statistiques des données Toutes les personnes enquêtées, appartenant à plusieurs groupes socioculturels (Abbey, Malinké, Attié, Appolo, ...), ont été réparties suivant le genre et la classe d'âge. La répartition suivant la classe d'âge a suivi celle d'Assogbadjo et al. (2008) comme suit: les jeunes (âge $\leq 30$ ans); les adultes (30 <âge $<60$ ); les vieux (âge $\geq 60$ ans). Du fait de la faible représentativité de certains groupes socioculturels (Attié, Appolo, Agni, Gouro, Guéré et les non nationaux) dans l'échantillon, pouvant biaiser les estimations, ces groupes socioculturels ont été fusionnés en «autres groupes socioculturels " (Tableau 1). Quatre indices ethnobotaniques: la fréquence relative de citation, la valeur d'utilisation totale rapportée, la valeur d'utilisation rapportée par l'organe et l'indice d'importance culturelle, ont été calculés pour évaluer respectivement les connaissances et le consensus des enquêtés sur ces connaissances, les usages et l'importance culturelle de $B$. vulgaris.

Tableau 1. Effectif des personnes enquêtées selon le groupe socioculturel, la classe d'âge et le genre.

Table 1. Number of people surveyed by socio-cultural group, age group and gender

\begin{tabular}{|llllllll|}
\hline Catégories socioculturelles & Abbey & & Malinkés & \multicolumn{3}{ll}{$\begin{array}{l}\text { Autres groupes } \\
\text { ethniques }\end{array}$} & Total \\
Genre & H & F & H & F & H & F & Total \\
Jeunes (âge $<30$ ans) & 19 & 12 & 16 & 10 & 23 & 17 & 97 \\
Adultes $(30<$ âge $<60)$ & 36 & 23 & 21 & 9 & 22 & 25 & 136 \\
Vieux (âge $\geq 60$ ans) & 10 & 3 & 3 & 2 & 3 & 4 & 25 \\
Total & 65 & 38 & 40 & 21 & 48 & 46 & 258 \\
\hline
\end{tabular}

H: Hommes; F: Femmes

La Fréquence Relative de Citation (FRC) se réfère au nombre de fois que les enquêtés d'un groupe social ont cité un usage spécifique donné, rapporté au nombre total des enquêtés de ce groupe (Assogba et al. 2017, Honfo et al. 2015, Tardio \& Pardo-De-Santayana 2008):

\section{$F R C=(n / N) \times 100$.}

Où FRC est la fréquence relative de la citation exprimée en pourcentage; $n$ le nombre d'enquêtés ayant cité un usage donné de $B$. vulgaris; $N$ le nombre total d'enquêtés. La FRC a permis d'ordonner les usages spécifiques des organes de $B$. vulgaris. Des valeurs élevées de la FRC pour un usage spécifique traduisent généralement un consensus pour cet usage de l'organe au sein de la communauté.

La valeur d'utilisation totale rapportée (VUR) (Gomez-Beloz 2002) pour un groupe est le nombre total moyen d'usages spécifiques rapporté pour $B$. vulgaris dans ce groupe, exprimé en usages spécifiques par enquêté:

$$
\text { VUR }=\left(\sum_{i=1}^{N} \mathrm{VURi}\right) / \mathrm{N}
$$

Où VURi est le nombre total d'usages spécifiques rapportés par l'enquêté i du groupe; $\mathrm{N}$ est le nombre total d'enquêtés pour le groupe social considéré. Cet indice permet de mesurer et de comparer les connaissances des enquêtés entre les différents groupes socioculturels, catégories d'âges et genres. Les groupes présentant des VUR élevées sont ceux possédant globalement plus de connaissances sur les usages de la plante.

Les valeurs de la VUR étant des données de comptage; elles ont été soumises à un modèle linéaire généralisé de la famille de Poisson pour tester l'effet du groupe socioculturel, de l'âge et du genre sur sa variation. Le modèle saturé (tous les effets principaux et interactions possibles) a été d'abord spécifié. Ensuite, une simplification du modèle a été opérée jusqu'à la sélection du modèle parcimonieux (Réduire le nombre possible de facteurs) (Johnson \& Omland 2004). Cette simplification a été basée sur la valeur du critère d'information d'Akaike corrigé pour les petites tailles d'échantillon (Akaike 1973). Les analyses ont été réalisées dans le logiciel $R$ version 3.4.3 ( $R$ Core Team 2016) et XLSAT.

La valeur d'utilisation rapportée par organe (VURorg) (Gomez-Beloz, 2002) est le nombre moyen d'usages rapportés pour chaque organe de 
B. vulgaris, exprimé en usages spécifiques par enquêté:

$$
\text { VURorg }=\left(\sum_{i=1}^{N} \text { VURix }\right) / \mathbf{N}
$$

Où VURorgx est la valeur d'utilisation rapportée pour l'organe; VURxi est le nombre total d'usages spécifiques de l'organe mentionné par l'enquêté du groupe considéré; $\mathrm{N}$ est le nombre total d'enquêtes du groupe. Cet indice a permis de tester le nombre d'usages connus par organe de la plante. Les organes des VURorg sont ceux qui ont le plus grand nombre d'usages, et donc probablement les plus souvent sollicités par les populations. Un modèle linéaire généralisé de Poisson utilisé pour tester la signification des différences des valeurs de VURorg entre organes de plante.

L'indice d'importance culturelle (IC) (Houehanou et al. 2011) définit, d'une part, la valeur culturelle allouée à la plante et, d'autre part, celle rattachée aux organes de la plante par les populations locales. La valeur de I'IC a été calculée par catégorie d'usages $\left(\mathrm{IC}_{\mathrm{CU}}\right)$ pour chaque groupe socioculturel d'une part et pour chaque organe de la plante d'autre part. Dans le premier cas, I'IC permet de mettre en exergue la catégorie d'usages la plus importante selon les groupes socioculturels et, dans le second cas, la catégorie d'usages la plus importante selon l'organe de la plante. La valeur d'IC a été par ailleurs calculée par organe ( $\mathrm{IC}_{\mathrm{Org}}$ ) et pour chaque groupe socioculturel pour mettre en exergue les les plus importants de la plante pour chaque groupe socioculturel.

Pour chaque groupe socioculturel (respectivement chaque organe), l'importance culturelle d'une catégorie d'usages quelconque $\mathrm{k}$ a été calculée en utilisant la formule ci-dessous:

$$
\text { ICcuk }=\left(\sum_{i=1}^{N} \text { SCUki }\right) / N
$$

Où $S_{\text {cuki }}$ est le score attribué par l'enquêté i du groupe socioculturel relié à la catégorie d'usages $\mathrm{k}$ $\left(\mathrm{CU}_{\mathrm{k}}\right)$ de la plante; $\mathrm{N}$ désigne le nombre total d'enquêtés.

L'indice d'importance culturelle de chaque organe de plante pour un groupe socioculturel donné a été calculé en utilisant la formule ci-dessous:

$$
\text { ICPP/Org }=\left(\sum_{i=1}^{N} \sum_{k=1}^{K} \text { SPP/Orgm }- \text { CUki }\right) / N
$$

Où Spp/Orgm-cuki est le score attribué par l'enquêté i du groupe socioculturel considéré à l'organe $m$ dans la catégorie d'usages $\mathrm{k}\left(\mathrm{CU}_{\mathrm{k}}\right)$ de la plante; $\mathrm{K}$ désigne le nombre total de catégories d'usages $(K=10$ dans la présente étude); $\mathrm{N}$ détermine le nombre total d'enquêtés du groupe socioculturel considéré. Les valeurs de IC étant des scores moyens, elles ont été soumises à un test de Kruskal-Wallis (Dytham 2011) pour l'importance de chaque catégorie d'usages de $B$. vulgaris entre groupes socioculturels et entre organes de la plante d'une part et d'autre part, l'importance culturelle de chaque organe de plante entre groupes socioculturels. Des diagrammes en radar ont été utilisés pour illustrer les résultats.

\section{Resultats}

\section{Populations enquêtées}

Le Tableau 1 renseigne l'effectif des populations interviewées en fonction de leurs catégories d'âge, du genre et de leurs groupes socioculturels. Au total, 258 personnes ont été interviewées avec une majorité d'adultes et d'autochtones Abbey.

\section{Diversité des connaissances et catégorisation des usages de $B$. vulgaris}

Le Tableau 2 présente la diversité des usages de $B$. vulgaris. II en ressort que les populations des villages de la sous-préfecture d'Azaguié connaissent en général 38 usages de l'espèce dont quelques-uns sont présentés àla Figure 2. En catégorisant ces usages l'on dénombre dix (10) domaines d'usages qui sont: la construction, l'alimentation, l'agriculture, la médecine, la culture, l'énergie, l'artisanat et le matériel, l'élevage, la pêche et l'écologie (Tableau 2 ). Les enquêtés ont révélé que l'espèce est plus utilisée dans le domaine de la construction (support pour échafaudage, clôture, hangar, claie, étagère, pont, charpente, etc) avec une Fréquence Relative de Citation (FRC) de 84,38\% de l'échantillon d'étude. Par contre, seulement 6,91\% ont rapporté l'usage de la plante dans l'alimentation. De plus, les usages dans les domaines de l'énergie (bois de chauffe) et de l'agriculture ont été mentionnés respectivement par $70,51 \%$ et $61,27 \%$ des enquêtés. Les usages en médecine, en élevage et dans le domaine de la pêche de $B$. vulgaris sont également non négligeables $(39,07 \%, 38,56 \%$ et $35,75 \%)$. $B$. vulgaris est utilisé en artisanat, dans le domaine socioculturel et d'autres fins utiles. L'usage dans le domaine écologique de $B$. vulgaris a été évoqué par $9,01 \%$ des enquêtés. II s'agit des branches et feuilles utilisées pour la confection des nids des tisserins à tête noire (Ploceus melanocephalus) (Figure 3) et aussi des chaumes désséchés qui deviennent le substrat des espèces de champignon telles que Neonothopanus hygrophanus. 
Tableau 2. Usages spécifiques de Bambusa vulgaris et leur importance (+++: très important; ++: moyennement important; +: peu important; -: pas cité) et fréquences de citation (FRC).

Table 2. Specific uses of $B$. vulgaris and their importance (+++: very important; ++: moderately important; + : not important ; - : not mentioned) and frequency of citation (RFC)

\begin{tabular}{|c|c|c|c|c|}
\hline $\begin{array}{l}\text { Catégories } \\
\text { d'usages }\end{array}$ & $\begin{array}{l}\text { Organe ou partie de la } \\
\text { plante }\end{array}$ & Usages spécifiques & Importance & FRC \\
\hline \multirow[t]{8}{*}{ Construction } & Chaumes & $\begin{array}{l}\text { Clôture de concessions ou } \\
\text { enclos }\end{array}$ & +++ & \\
\hline & & Maison (échafaudage) & +++ & \\
\hline & & Hangar pour petits commerces & +++ & $80,36 \%$ \\
\hline & & Toiture de maison & ++ & \\
\hline & & Claie pour sécher les récoltes & ++ & \\
\hline & & Pont de fortune pour le passage & ++ & \\
\hline & & $\begin{array}{l}\text { Etagère à l'allure de table au } \\
\text { marché }\end{array}$ & ++ & \\
\hline & & Préaux pour cuisine ou repos & +++ & \\
\hline \multirow[t]{3}{*}{ Alimentation } & Pousses (tiges) & Légumes pour la cuisine & + & \\
\hline & & Boisson (bière) & - & \\
\hline & Feuilles eng. & Extraction du vin de palme & + & $6,91 \%$ \\
\hline Matériel et & Chaumes & Ustensiles & + & \\
\hline \multirow[t]{5}{*}{ Artisanat } & & Mobilier & + & \\
\hline & & Manches d'outils & + & \\
\hline & & Objets décoratifs & + & $20,27 \%$ \\
\hline & Branches+chaumes & Objets décoratifs & + & \\
\hline & & Ustensiles & + & \\
\hline \multirow[t]{4}{*}{ Médecine } & Feuilles & Fièvre typhoïde & +++ & \\
\hline & & Paludisme & + & \\
\hline & Racines & Diarrhée infantile & ++ & $39,07 \%$ \\
\hline & & Accouchement difficile & + & \\
\hline \multirow[t]{2}{*}{ Pêche } & chaumes (fibres) & Nasse pour la pêche & + & \\
\hline & Branches & Canne à pêche & ++ & $35,75 \%$ \\
\hline \multirow[t]{3}{*}{ Elevage } & Chaumes & Mangeoire & + & \\
\hline & & Fourrage pour bétails & ++ & $38,56 \%$ \\
\hline & & Abreuvoir & + & \\
\hline \multirow[t]{3}{*}{ Agriculture } & Chaumes+branches & Tuteur pour les plantes & +++ & \\
\hline & & Ombrière & ++ & $61,27 \%$ \\
\hline & & Support de pépinière & ++ & \\
\hline \multirow[t]{2}{*}{ bois énergie } & Chaumes & Bois de chauffe & +++ & $70,51 \%$ \\
\hline & & Charbon de bois & + & \\
\hline \multirow[t]{2}{*}{ Socioculturel } & Branches & Instrument de musique & + & \\
\hline & Chaumes & $\begin{array}{l}\text { Fourches de soutien de certains } \\
\text { fétiches }\end{array}$ & ++ & $13,85 \%$ \\
\hline \multirow[t]{3}{*}{ Ecologie } & Chaumes & $\begin{array}{l}\text { Substrat d'une espèce de } \\
\text { champignon }\end{array}$ & + & \\
\hline & Branches & Nids de tisserins & + & $9,01 \%$ \\
\hline & Feuilles & Nids de tisserins & ++ & \\
\hline \multirow[t]{4}{*}{ Autres usages } & Chaumes & Sèche-linge & + & \\
\hline & & Poteaux électriques & ++ & $40,38 \%$ \\
\hline & & Poteaux de football & + & \\
\hline & & Jouets pour enfants & + & \\
\hline
\end{tabular}




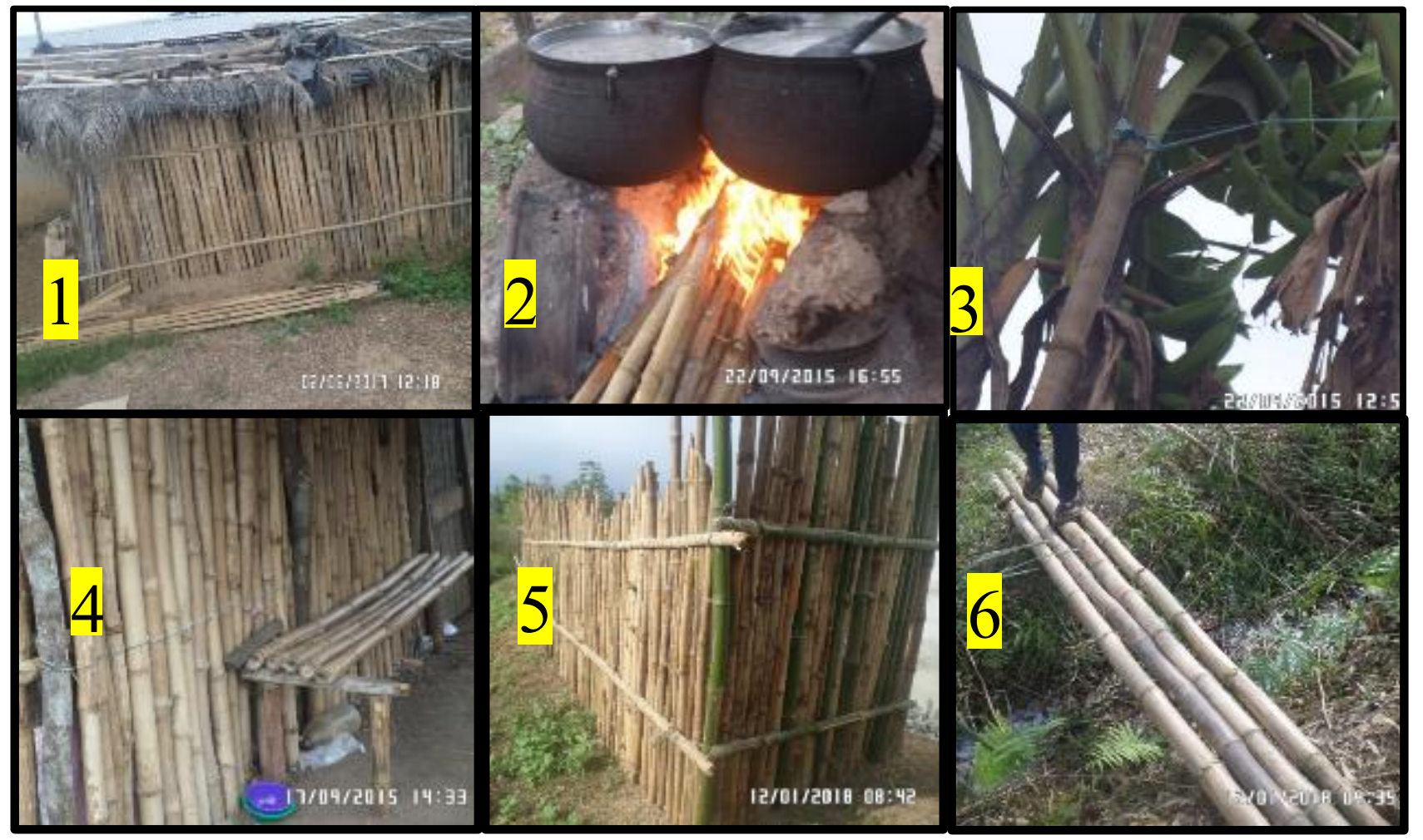

Figure 2. Quelques usages de Bambusa vulgaris dans la sous-préfecture d'Azaguié: 1) Maison en bambou; 2) Bambou comme bois de chauffe; 3) Bambou comme support; 4) Banc fait en bambou; 5) Clôture en bambou; 6) Pont de fortune en bambou.

Figure 2. Some uses of Bambusa vulgaris in Azaguie sub-prefecture: 1) B. vulgaris house; 2) B. vulgaris as firewood; 3) B. vulgaris as a support; 4) Bench made of $B$. vulgaris; 5) B. vulgaris fence; 6) B. vulgaris makeshift bridge.

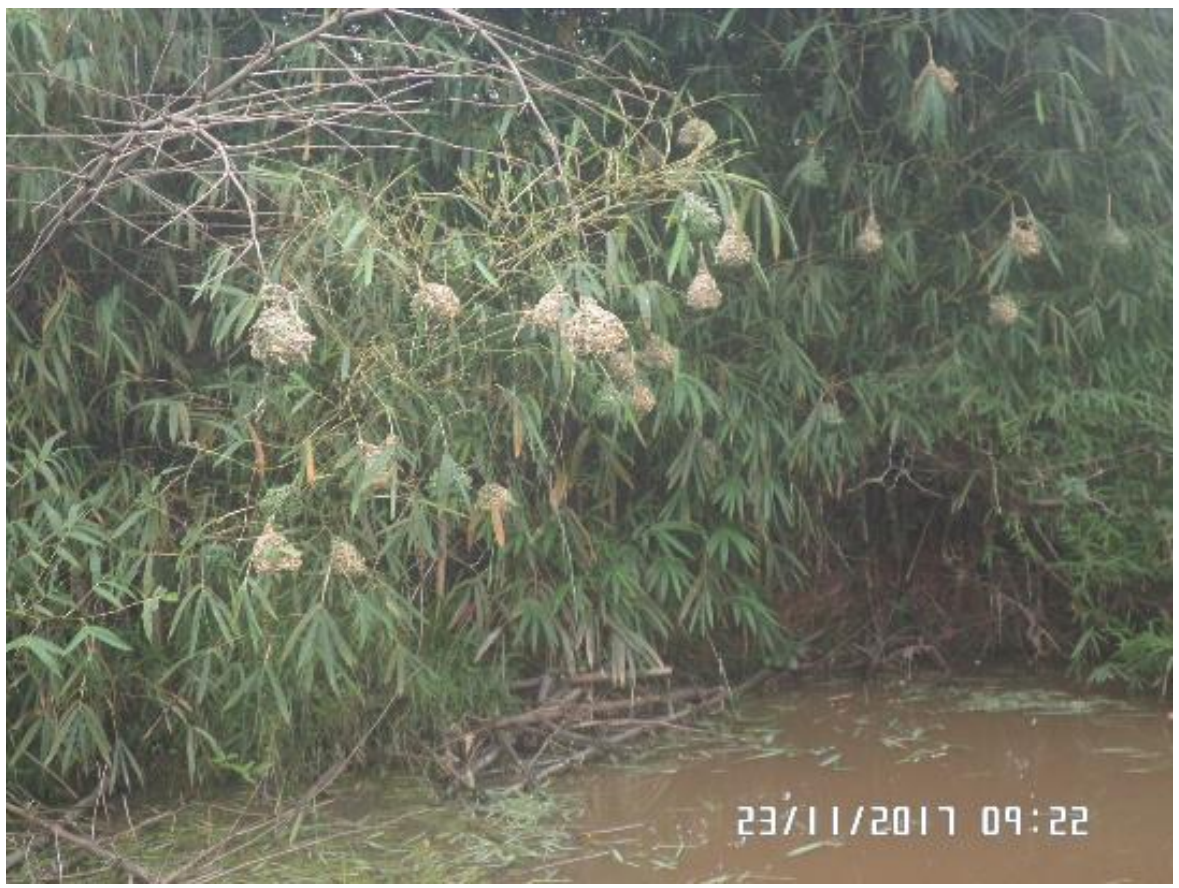

Figure 3. Vue photographique des nids de tisserins sur des rameaux feuillés de $B$. vulgaris.

Figure 3. Photographic view of weaver nests on leafy twigs of $B$. vulgaris. 
En répartissant ces Fréquences Relatives de Citation (FRC) en fonction des différents villages (Tableau 3 ) et en considérant les quatre principaux domaines d'utilisation, l'on remarque qu'à AzaguiéAhoua, le bambou est plus utilisé dans la construction $(84,80 \%)$ et l'énergie $(78,18 \%)$.
M'Bromé s'illustre plus avec l'usage agricole $(79,41)$, tandis qu'à Azaguié-Gare l'on s'intéresse moins à l'usage médicinal $(20,94 \%)$. En dehors de ces extrêmes, toutes les valeurs des FRC de ces quatre principaux usages dans les villages sont plus ou moins similaires.

Tableau 3. Variations des fréquences relatives de citations par villages

Table 3. Variations in relative frequencies of citation by villages

\begin{tabular}{lllll} 
& & & & \\
\cline { 2 - 5 } Catégories d'usages & Abbè-Béguini & Azaguié-Ahoua & Azaguié-Gare & M'Bromé \\
\cline { 2 - 5 } Construction & 77,05 & 84,8 & 64,47 & 65,12 \\
\hline Alimentation & 6,06 & 2,72 & 8,1 & 10,78 \\
\hline Agriculture & 52,52 & 60 & 53,15 & 79,41 \\
\hline Médecine & 32,57 & 30,45 & 20,94 & 32,35 \\
\hline Culture & 17,17 & 16,36 & 7,2 & 61,76 \\
\hline Energie & 77,27 & 78,18 & 64,86 & 19,11 \\
\hline Artisanat & 11,36 & 18,18 & 32,43 & 29,41 \\
\hline Elevage & 32,52 & 50,9 & 41,44 & 54,41 \\
\hline Pêche & 37,87 & 34,54 & 16,21 & 45,58 \\
\hline Autres & 44,69 & 38,18 & 33,1 & \\
\hline
\end{tabular}

Facteurs socio-démographiques influençant le niveau de connaissance des usages de $B$. vulgaris

Le Tableau 4 résume les différents tests de KruskalWallis effectués pour montrer l'influence du niveau de connaissances sur les usages. Parmi les facteurs testés, seul l'âge détermine les variations du niveau de connaissances des usages de $B$. vulgaris. En effet, la valeur de connaissance des usages de $B$. vulgaris chez les vieillards est significativement plus élevé $(6,80 \pm 0,10)$ que celle des adultes $(3,93 \pm$ $0,05)$ et des jeunes $(3,37 \pm 0,10)$ avec $P=0,001$. Par contre, il n'y a pas de différence significative entre les connaissances des adultes et celles des jeunes. Les facteurs " genre " et " groupe socioculturel » n'enregistre aucune différence significative entre les variables $(p=0,791$ et $p=0,747)$. La valeur de connaissance des usages de $B$. vulgaris est pratiquement similaire entre les Abbeys (4,70 \pm $0,24)$, les Malinkés $(4,32 \pm 0,21)$ et les autres groupes socioculturels $(3,92 \pm 0,19)$. II en est de même entre les hommes $(4,27 \pm 0,18)$ et les femmes $(4,36 \pm 0,23)$ en ce qui concerne le facteur genre.

Tableau 4. Variation de la valeur de connaissance des usages de $B$. vulgaris des enquêtés: résultats de la sélection des facteurs socio-démographiques (âge, genre, groupe socioculturel).

Table 4. Variation in the knowledge value of respondents' $B$. vulgaris uses: results of socio-demographic factors selection (age, gender, socio-cultural group).

\begin{tabular}{|c|c|c|c|c|c|c|}
\hline Facteurs & Variables & Moyenne & Ecart-type & VCU & $\begin{array}{l}\text { Statistique } \\
\text { de test }\end{array}$ & $p$-value \\
\hline \multirow{3}{*}{ Age } & Jeunes & 2,20 & 0,03 & $2,20 \pm 0,03^{a}$ & \multirow{3}{*}{$\mathrm{K}=15,17$} & \multirow{3}{*}{0,001} \\
\hline & Adultes & 3,93 & 0,05 & $3,93 \pm 0,05^{a b}$ & & \\
\hline & Vieux & 6,80 & 0,10 & $6,80 \pm 0,10^{b}$ & & \\
\hline \multirow{3}{*}{$\begin{array}{l}\text { Groupe } \\
\text { socioculturel }\end{array}$} & Abbeys & 4,70 & 0,24 & $4,70 \pm 0,24^{c}$ & \multirow{3}{*}{$K=0,58$} & \multirow{3}{*}{0,747} \\
\hline & Malinkés & 4,32 & 0,21 & $4,32 \pm 0,21^{\mathrm{c}}$ & & \\
\hline & Autres & 3,92 & 0,19 & $3,92 \pm 0,19^{c}$ & & \\
\hline \multirow[t]{2}{*}{ Genre } & Hommes & 4,27 & 0,18 & $4,27 \pm 0,18^{d}$ & \multirow[t]{2}{*}{$\mathrm{K}=0,07$} & \multirow[t]{2}{*}{0,791} \\
\hline & Femmes & 4,36 & 0,23 & $4,36 \pm 0,23^{d}$ & & \\
\hline
\end{tabular}

Les VCU affectées de la même lettre ne sont significativement différentes au seuil $5 \%$.

Valeur d'usage totale rapportée (VUR) de Bambusa vulgaris

Les moyennes des différents usages spécifiques rapportés par village sont regroupées sous le vocable de valeur d'usage totale rapportée (VUR).
Ainsi, les populations d'Azaguié-Ahoua montrent plus de connaissances sur les usages faits de Bambusa vulgaris avec une moyenne de 14,21. Celles d'Azaguié-Gare ont peu de connaissances sur les usages (VUR=11,40). Les habitants de Abbè- 
Béguini et ceux de M'Bromé présentent des niveaux de connaissances intermédiaires avec respectivement des valeurs de VUR de 13,57 et 13,67 (Figure 4).

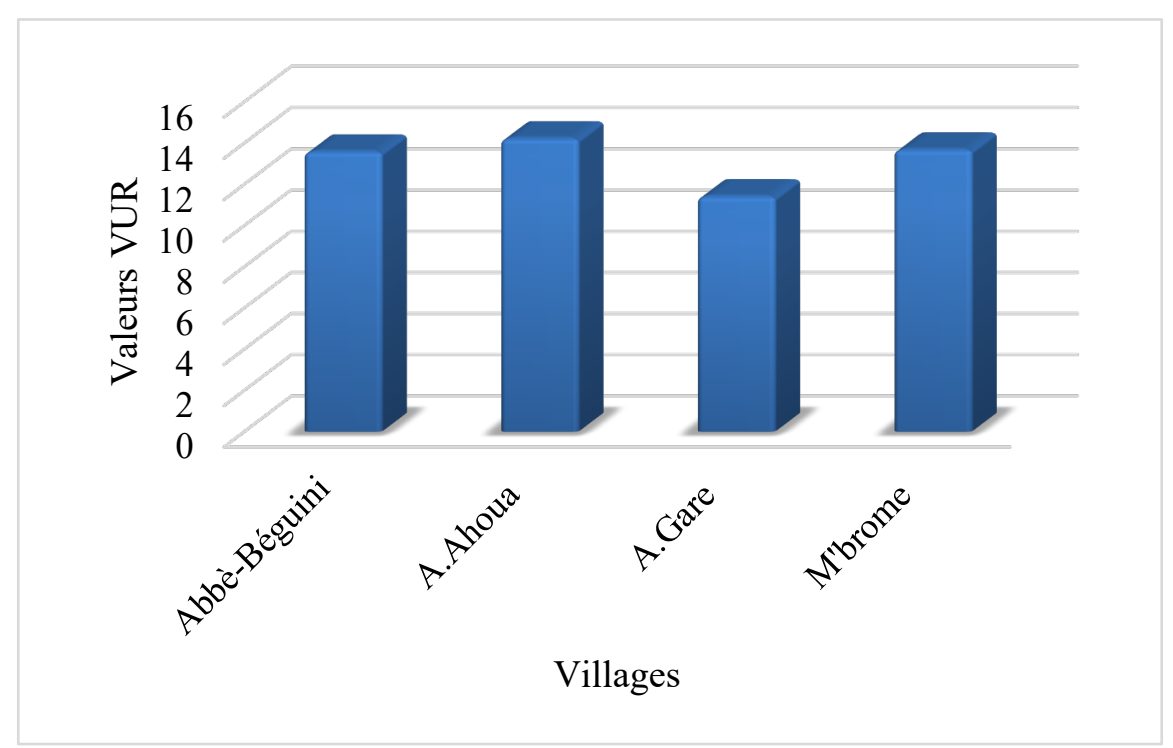

Figure 4. Histogrammes de distribution des différentes Valeurs d'usage totales rapportées de $B$. vulgaris par village. (A. Ahoua=Azaguié-Ahoua; A. Gare=Azaguié-Gare).

Figure 4. Histograms distribution of different reported total use values of $B$. vulgaris by village.

Valeur d'usage par organes de Bambusa vulgaris Différents organes de Bambusa vulgaris sont utilisés dans la Sous-préfecture d'Azaguié (Figure 5). La valeur d'usage par organe de $B$. vulgaris varie sensiblement entre le chaume et les autres organes de la plante. Le chaume $(0,35 \pm 0,32)$ est l'organe le plus utilisé, suivi de la feuille $(0,07 \pm 0,14)$ et de la branche $(0,06 \pm 0,17)$ (Figure 6). Les organes les moins utilisés sont la feuille engainante ou bractée et les racines $(0,02 \pm 0,07)$. Quant à la pousse elle n'a pas été mentionnée dans les différents usages spécifiques de $B$. vulgaris.

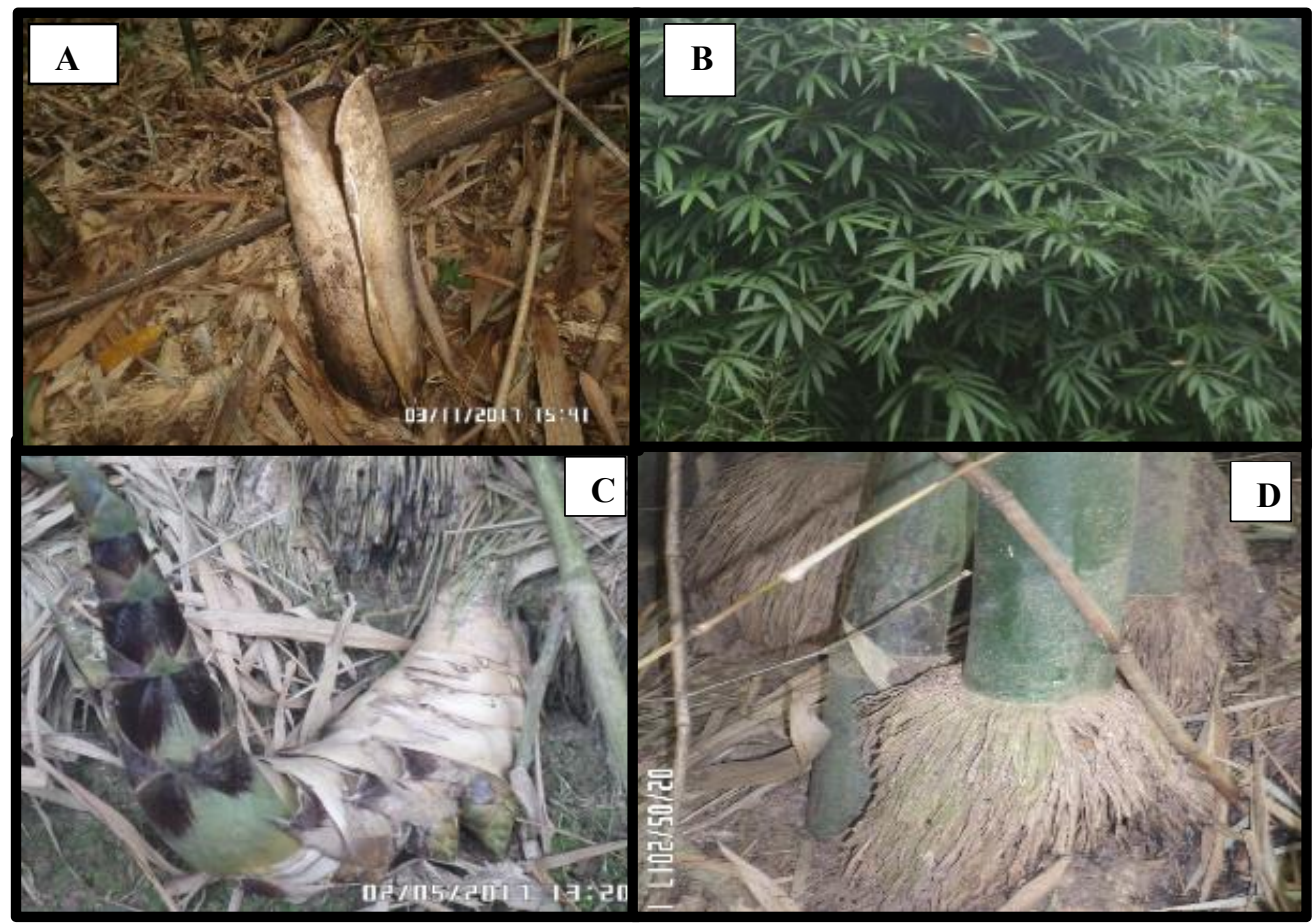

Figure 5. Quelques organs ou parties de Bambusa vulgaris utilizes dans la sous-préfecture d'Azaguie: A. Bractée ou feuille engainante; B) Feuilles classiqes; C) Jeune pousse ou turion; D) Chaume et Racines.

Figure 5. Some organs or parts of B. vulgaris used in Azaguie sub-prefecture: A) Bract or foil ; B) Leaves ; C) Shoot; D) Thatch and Roots. 


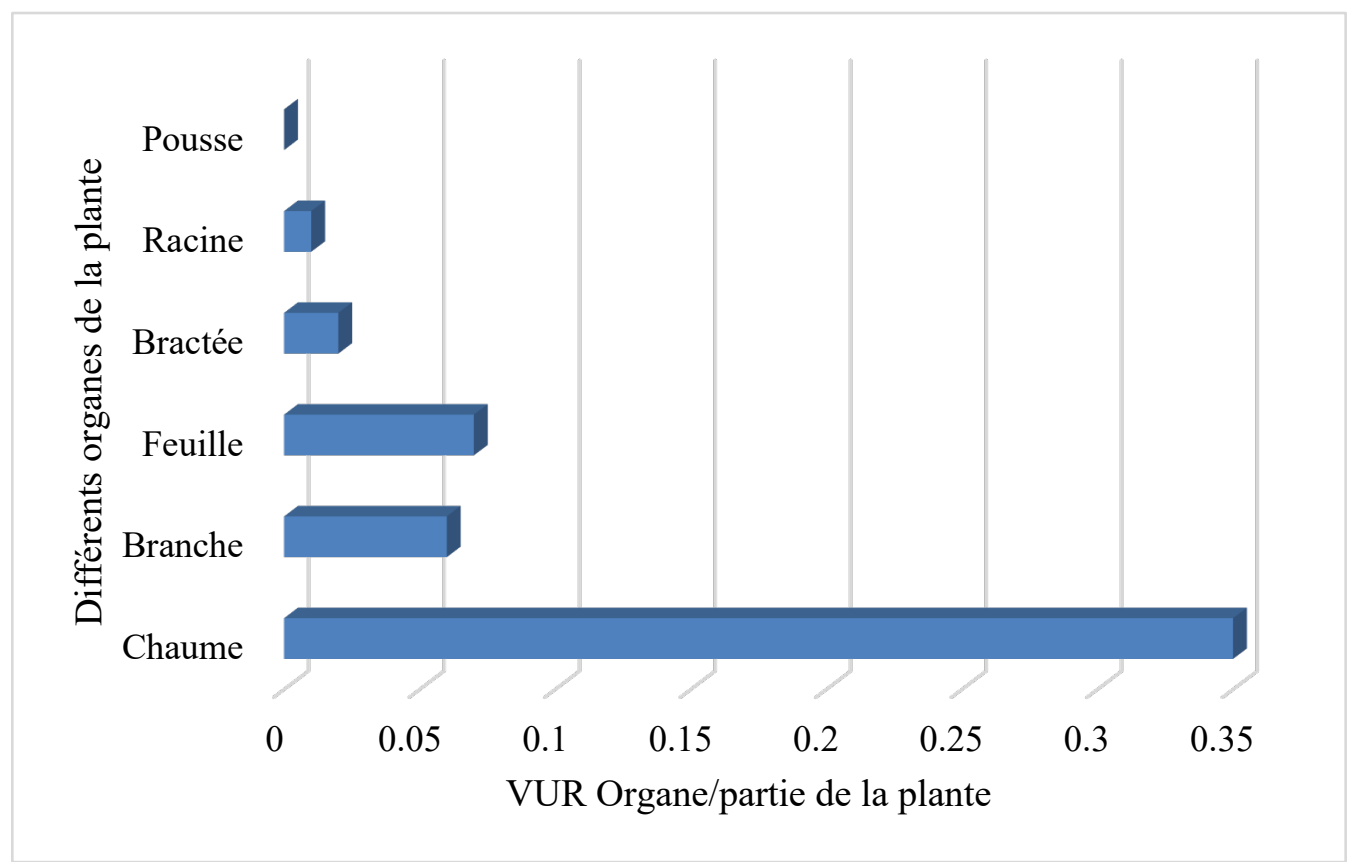

Figure 6. Diagramme en barre de la valeur d'usage des organes de Bambusa vulgaris.

Figure 6. Bar graphs of the use values of the organs of $B$. vulgaris

Importance culturelle des organes de $B$. vulgaris L'importance culturelle de chaque organe de $B$. vulgaris diffère significativement entre les catégories d'usages (Tableau 5, Figure 7). Cependant, l'importance culturelle du chaume, de la feuille et de la branche ne diffère pas entre les villages. En effet, le chaume est culturellement plus important pour les usages dans la construction, en agriculture et comme bois de chauffe. La feuille et la branche sont importantes pour les usages médicinaux, l'élevage (fourrage), la pêche et aussi pour ses avantages écologiques. La bractée et la jeune pousse (turion) sont plus sollicitées pour les usages alimentaires.
La Figure 8 présente l'importance culturelle des organes de $B$. vulgaris en fonction des villages. Le chaume a une importance culturelle plus grande pour les villages alors que la feuille est plus importante pour les populations de Abbè-Béguini et celles de M'Bromé. Contrairement aux autres villages la bractée est non négligeable à AbbèBéguini et aussi la racine fait partie des préférences des populations Abbè-Béguini pour la médecine traditionnelle (Figure 8).

Tableau 5. Variation de l'importance culturelle des catégories d'usages de $B$. vulgaris entre les différents villages: résultats du test de Kruskal-Wallis.

Table 5. Variation of the cultural importance of B.vulgaris use categories between different villages

\begin{tabular}{lllll}
\hline Catégories & Abbè-Béguini & Azaguié-Ahoua & Azaguié-Gare & M'Bromé \\
\hline Construction & $0,76 \pm 0,13^{\mathrm{a}}$ & $0,87 \pm 0,16^{\mathrm{a}}$ & $0,65 \pm 0,12^{\mathrm{a}}$ & $0,67 \pm 0,25^{\mathrm{a}}$ \\
\hline Alimentation & $0,10 \pm 0,18^{\mathrm{a}}$ & $0,06 \pm 0,11^{\mathrm{b}}$ & $0,09 \pm 0,06^{\mathrm{a}}$ & $0,13 \pm 0,22^{\mathrm{a}}$ \\
\hline Agriculture & $0,58 \pm 0,27^{\mathrm{a}}$ & $0,50 \pm 0,25^{\mathrm{ab}}$ & $0,55 \pm 0,34^{\mathrm{a}}$ & $0,71 \pm 0,20^{\mathrm{a}}$ \\
\hline Energie & $0,39 \pm 0,53^{\mathrm{a}}$ & $0,52 \pm 0,64^{\mathrm{ab}}$ & $0,58 \pm 0,31^{\mathrm{a}}$ & $0,36 \pm 0,43^{\mathrm{a}}$ \\
\hline Médecine & $0,42 \pm 0,49^{\mathrm{a}}$ & $0,44 \pm 0,35^{\mathrm{ab}}$ & $0,41 \pm 0,52^{\mathrm{a}}$ & $0,52 \pm 0,40^{\mathrm{a}}$ \\
\hline Socioculturel & $0,19 \pm 0,15^{\mathrm{a}}$ & $0,20 \pm 0,08^{\mathrm{ab}}$ & $0,10 \pm 0,10^{\mathrm{a}}$ & $0,24 \pm 0,16^{\mathrm{a}}$ \\
\hline Pêche & $0,45 \pm 0,12^{\mathrm{a}}$ & $0,37 \pm 0,12^{\mathrm{ab}}$ & $0,11 \pm 0,09^{\mathrm{a}}$ & $0,30 \pm 0,08^{\mathrm{a}}$ \\
\hline Artisanat & $0,10 \pm 0,02^{\mathrm{a}}$ & $0,13 \pm 0,01^{\mathrm{ab}}$ & $0,34 \pm 0,02^{\mathrm{a}}$ & $0,22 \pm 0,19^{\mathrm{a}}$ \\
\hline Elevage & $0,33 \pm 0,14^{\mathrm{a}}$ & $0,35 \pm 0,09^{\mathrm{ab}}$ & $0,42 \pm 0,13^{\mathrm{a}}$ & $0,35 \pm 0,05^{\mathrm{a}}$ \\
\hline Ecologie & $0,32 \pm 0,21^{\mathrm{a}}$ & $0,21 \pm 0,28^{\mathrm{ab}}$ & $0,16 \pm 0,15^{\mathrm{a}}$ & $0,28 \pm 0,16^{\mathrm{a}}$ \\
\hline Autres & $0,41 \pm 0,29^{\mathrm{a}}$ & $0,45 \pm 0,37^{\mathrm{ab}}$ & $0,33 \pm 0,25^{\mathrm{a}}$ & $0,51 \pm 0,25^{\mathrm{a}}$ \\
\hline Kruskal-Wallis & 14,04 & 16,31 & 14,84 & 13,45 \\
\hline p-value & 0,081 & 0,038 & 0,062 & 0,097
\end{tabular}

Les ICU affectées de la même lettre ne sont significativement différentes au seuil $5 \%$. 


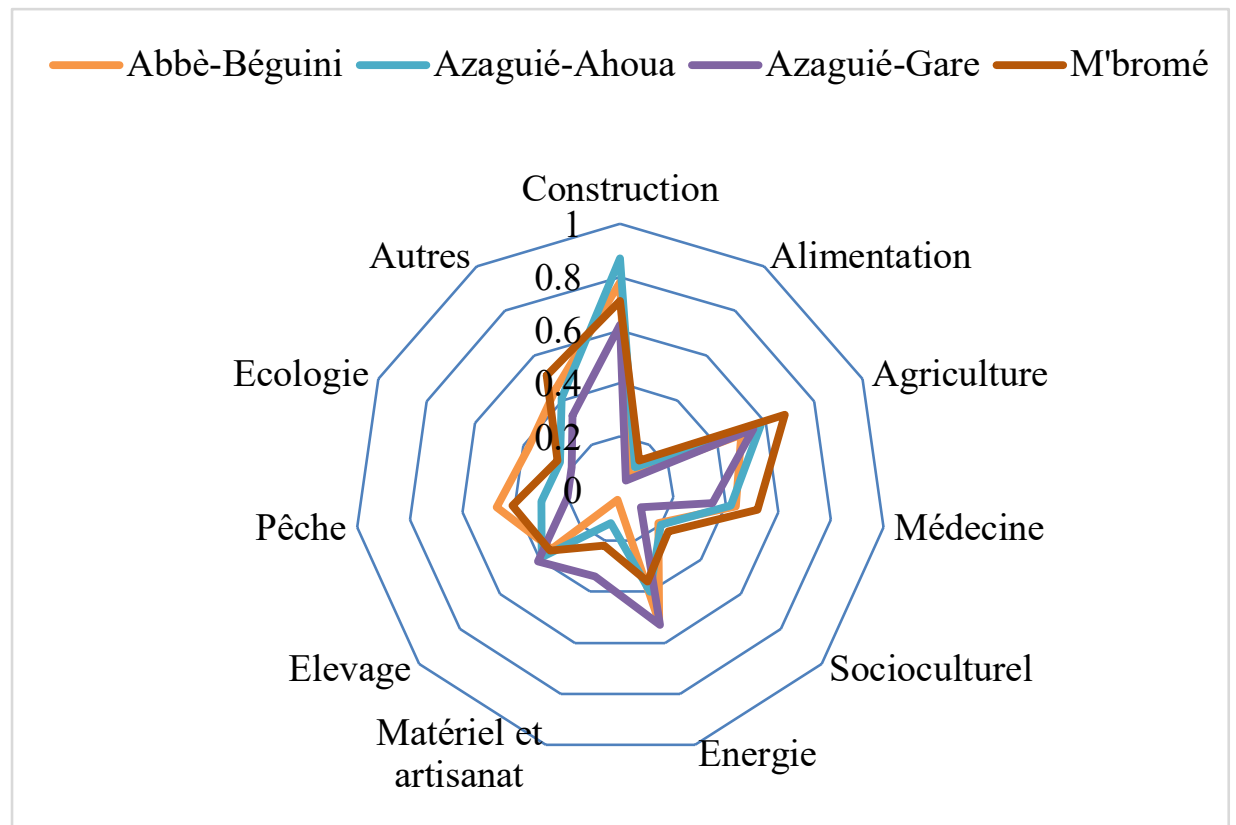

Figure 7. Diagramme en radar décrivant l'importance culturelle de Bambusa vulgaris en fonction des villages. Figure 7. Radar diagram depicting cultural importance of $B$. vulgaris by villages.

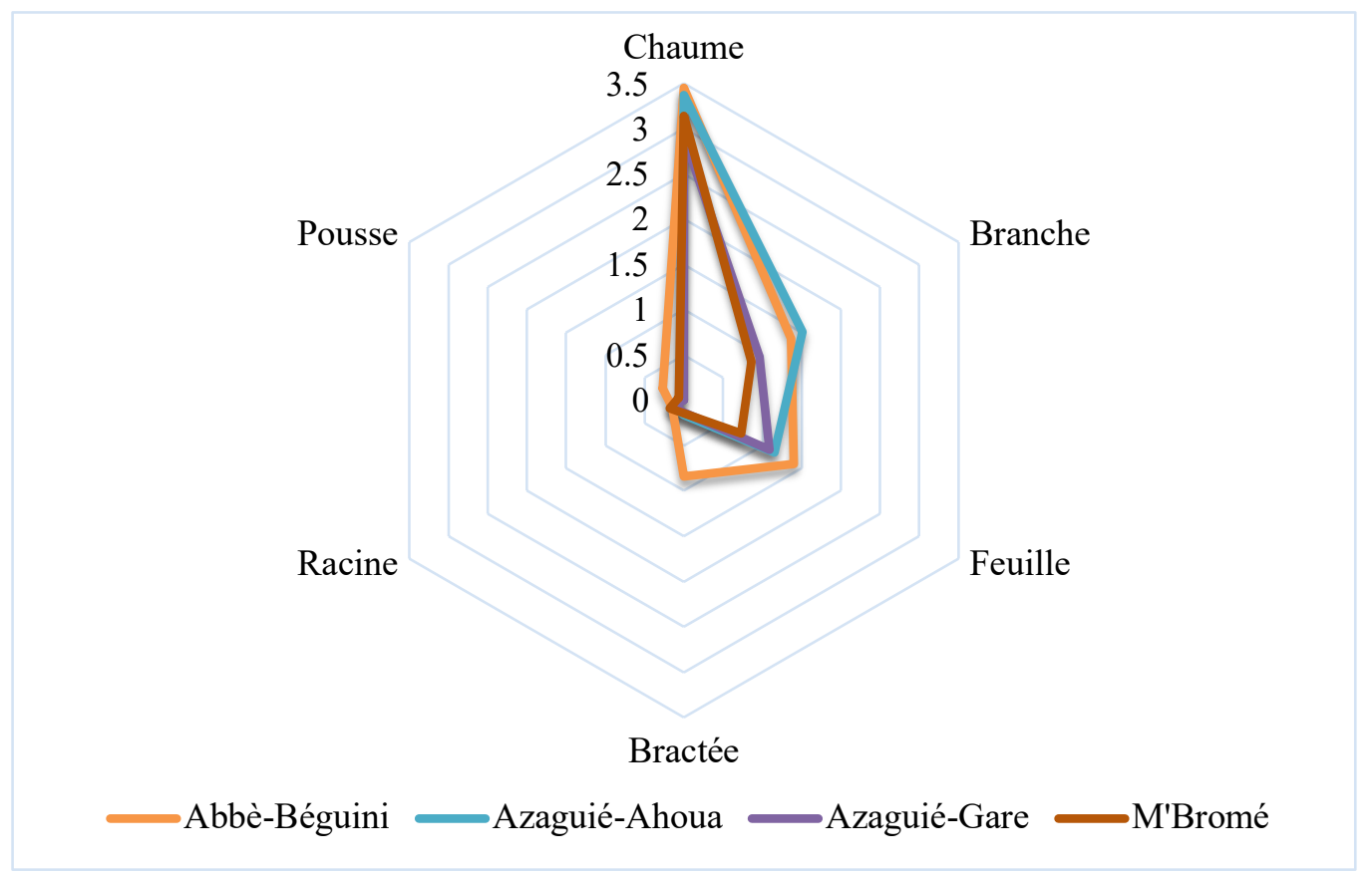

Figure 8. Diagramme en radar décrivant l'importance culturelle des organes de Bambusa vulgaris en fonction des villages.

Figure 8. Radar diagram depicting cultural importance of $B$. vulgaris organs in different villages.

\section{Discussion}

Connaissances diversifiées des catégories d'usages de $B$. vulgaris

Cette étude sur $B$. vulgaris, l'une des rares du genre en Côte d'Ivoire, est une contribution à la meilleure connaissance du PFNL et de son potentiel pour l'amélioration des conditions de vie des populations rurales. Cela confirme les résultats des travaux de Dje et al. (2017) et ceux de Zadou et al. (2011). Chaque groupe socioculturel, en dehors des autochtones Abbey, ne désigne pas $B$. vulgaris par une appellation spécifique. Tout le monde l'appelle couramment «bambou de Chine » et chez les Abbey seuls les vieillards et quelques adultes le désignent par « Ohojo ». Ceci révèle, le contact et la tradition récente de l'espèce dans l'histoire des peuples vivants dans la sous-préfecture d'Azaguié. La plante étant exogène donc il est difficile de lui trouver une appellation idoine. II n'existe donc pas de différences dans les appellations par groupe socioculturel. Cela 
n'est pas toujours le cas pour tous les PFNL, car les travaux de Gruca et al. (2015) sur Borassus aethiopum au Ghana et de Assogba et al. (2017) sur Bombax costatum au Benin ont montré des différences d'appellation dans les différents groupes socioculturels. Dans le cas présent, il n'est pas évident que l'appellation ait un lien avec l'usage préférentiel de l'espèce pour chaque groupe sociolinguistique. Au total, l'étude a permis de recenser 38 usages de l'espèce, classés en dix catégories d'usages, à savoir: construction, alimentation, agriculture, médecine, culture, énergie, artisanat et matériel, élevage, pêche et écologie. Ces résultats montrent une grande diversité des usages de l'espèce dans les villages de la sous-préfecture d'Azaguié. Les usages multiples de $B$. vulgaris sont quasiment semblables à ceux du palmier (Rainer et al. 2015) et du baobab (Rashford, 2015). Les catégories d'usages les plus importantes sont la construction, l'énergie, l'agriculture et celle de la médecine. Cela s'expliquerait par le fait que dans la zone d'Azaguié, avec l'avènement des cultures agroindustrielles, les forêts villageoises ont presque disparu. Les bois d'œuvre sont devenus denrées rares. N'ayant pas un accès libre à la forêt classée de Yapo-Abbé, l'on assiste donc à une ruée vers le bambou pour l'utiliser dans le domaine de la construction et comme bois de chauffe. En agriculture, il est apprécié pour sa dureté et sa résistance. C'est pour cette raison qu'il est utilisé comme tuteur des papayers, des fruits de la passion, comme soutien pour les régimes de banane et pour la confection des ombrières des pépinières. Les travaux de Nath et al. (2011) dans la vallée de Barak au Nord-Est de I'Inde par contre ont montré que l'usage alimentaire était le plus prépondérant, suivi de l'usage dans la construction. Dans cette partie du monde et aussi en Asie orientale (Karanjal et al. 2015, Nirmala et al. 2014), les pousses (turions) des espèces de bambou telles que Bambusa balcooa et Bambusa vulgaris étaient très prisées dans l'alimentation en tant que légumes. Les chaumes étaient majoritairement destinés à la construction dans les villages d'Azaguié-Ahoua et Abbè-Béguini en ce sens que la fréquence de construction des habitats modernes dans ces localités devient de plus en plus élevée. L'autre raison qui pourrait expliquer cette situation est la présence de la majorité des acteurs de la filière. Tesfaye et al. (2011) justifient l'usage intense du bambou à Addis-Abeba en faisant référence au nombre élevé de commerçants qu'on y trouve. Quant à Babulo et al. (2009), ils relient l'usage intensif à la commercialisation régulière. L'illustration du village de M'Bromé avec l'usage des chaumes en agriculture s'expliquerait par le fait c'est une zone dont l'activité principale de population est l'agriculture. L'on ne manque d'y trouver des plantations agro-industrielles dont certaines nécessitant des chaumes de $B$. vulgaris pour la confection d'ombrière de pépinières ou comme tuteur de certaines plantes. Les jeunes plants de Passiflora eudilis Sims (Passifloraceae) se servent des chaumes et branches de $B$. vulgaris pour leur expansion (Dje Bi et al. 2017). Les chaumes de bambou à Azaguié-Gare sont surtout utilisés comme bois de chauffe. En effet, la majorité des habitants de ce village sont des allogènes burkinabés et des allochtones. De ce fait, l'usage intensif du $B$. vulgaris comme source d'énergie serait lié à la fabrication d'une boisson traditionnelle, le « Tchapalo ».

Impact des facteurs socio-démographiques sur le niveau de connaissance des usages de $B$. vulgaris

II n'existe pas de variation significative de connaissances dans l'utilisation de l'espèce entre groupes socioculturels et les genres de chaque peuple. Ce résultat pourrait s'expliquer par le fait que l'espèce Bambusa vulgaris (exotique) est introduite en Côte d'lvoire et ses usages ne sont pas spécifiques à un peuple. Toutefois, c'est au niveau de l'âge qu'on a observé une certaine variation du niveau de connaissance. La classe des vieillards était celle qui possédait le plus de connaissance sur les différents usages. Ce résultat pourrait être expliqué par le fait que dans une communauté, les plus âgés sont le plus souvent dépositaires de savoirs. Ces résultats sont en accord avec ceux de Bitariho \& Mcneilage (2008) en Ouganda, Creswell (2003), Fayera et al. (2016), Vedeld et al. (2007), en Ethiopie sur les niveaux de connaissances des usages de bambou. Dans d'autres régions du pays l'usage de $B$. vulgaris est remplacé par d'autres espèces plus répandues notamment dans la construction et la production de charbon (Gnahoua et al. 2014). C'est à Azaguié-Ahoua que la moyenne d'usage spécifique rapporté était la plus élevée. Cela est non seulement en rapport avec les populations qu'on y trouve mais aussi lié au nombre d'activités socio-économiques exercées dans le village. Certaines activités nécessitent l'usage permanent des chaumes et d'autre l'usage occasionnel. II n'était pas rare de voir $B$. vulgaris entrer dans la fabrication des sièges de kiosques à café ou de gargotes. Aussi les petites bâtisses (claie, hangar, poteaux de football, etc) sont-elles faites avec des chaumes souvent fendus en lamelles. II n'y avait pas grande variation dans les catégories d'usages d'un village à un autre sauf au niveau de l'usage dans la construction. Dans tous les villages, presque tous les usages spécifiques de la construction étaient observés ou mentionnés. Cet état des faits serait dû à l'importance culturelle de cette espèce surtout dans ce domaine d'activités. Mukul \& Rana (2013), dans leur étude sur les usages du bambou au Bengladesh, ont montré que le lien culturel entre la 
ressource et les communautés les poussaient à en faire plus d'usages. Cela reviendrait à dire que plus la ressource est plus proche d'un peuple plus ils en font un usage prioritaire.

\section{Conclusion}

Cette étude ethnobotanique de $B$. vulgaris au sein des groupes socioculturels vivant dans la souspréfecture d'Azaguié confirme l'importance de l'intégration de la culture de l'espèce dans les espaces villageois pour une diversification des usages et la conservation de la biodiversité. Bien que l'espèce soit utilisée par tous les groupes socioculturels, elle demeure sous-valorisée (exploitation informelle et peu d'investissement) sur le marché local malgré ses atouts dans la construction, l'énergie et l'agriculture. L'importance de l'usage des chaumes dans la construction indique un potentiel de l'espèce dans les stratégies d'aménagement des espaces basées sur les ressources locales. De même, le fort potentiel de l'espèce en artisanat traditionnel suggère la valorisation de ce secteur en vue d'une probable augmentation de la valeur ajoutée de la ressource et une exploitation conséquente. Ceci pourrait être une source de revenus pour les ménages ruraux. Nous suggérons donc que cette étude soit étendue à d'autres localités d'occurrence de l'espèce et au sein d'autres groupes socioculturels, afin d'identifier d'autres utilisations possibles de l'espèce pour une meilleure valorisation de ses potentialités. L'évaluation de la valeur commerciale des chaumes, des revenus générés par cette commercialisation et de l'identification des acteurs d'une filière de commercialisation est également capitale pour la valorisation de la plante. Les études antérieures de propagation entamées devront également être poursuivies en vue de la maîtrise de la culture de l'espèce et l'introduction de nouveaux genres de bambou.

\section{Déclarations}

Liste des abréviations:

CSRS: Centre Suisse de Recherche Scientifique DEIF: Direction des Exploitations et Industries Forestières

FAO: Fonds des Nations Unies pour l'Agriculture

FMTE: Foret des Marais

Tanoe-Ehy

FRC: Fréquence Relative de Citation

IC: Importance Culturelle

ODD: Objectifs de Développement Durable

PASRES: Programme d'Appui Stratégique à la

Recherche Scientifique

PFNL: Produits Forestiers Non Ligneux

RGPH: Recensement Général de la Population et de l'Habitat

VUR: Valeur d'Usage Relative
Approbation éthique et consentement à participer: Toutes les personnes interviewées étaient totalement consentantes et ont donné leur accord pour participer aux enquêtes.

Consentement pour la publication: Toute personne figurant sur un image qui sera publiée dans ce travail a donné son accord pour cela. Aucune image n'est publiée ici sans l'aval de l'individu concerné.

Disponibilité des données et des matériaux: Les données collectées et traitées dans le cadre du présent sont disponibles auprès des auteurs et pourraient les apporter si la revue manifeste la demande

Financement: Exemple: Cette étude a été financée par le Centre Suisse de Recherche Scientifique en Cote d'Ivoire (CSRS) a apporté le premier financement de cette étude.

Conflit d'intérêts: Les auteurs de ce manuscrit déclarent qu'ils n'ont aucun conflit d'intérêts.

Contributions des auteurs: Dans la réalisation de la présente étude, BDPVD et JKK ont élaboré le protocole de recherche, collecté les données, les ont traité et rédigé le manuscrit. JKK a participé à la relecture et au recadrage du document. De plus, il a peaufiné les textes du manuscrit. En outre, YCYA a supervisé toutes les activités depuis la recherche de financement en passant par la conception du protocole de recherche pour aboutir au document final. II a apporté des conseils dans la collecte et le traitement des données et surtout dans la rédaction du document.

\section{Remerciements}

Les auteurs remercient le Centre Suisse de Recherche Scientifique en Côte d'Ivoire (CSRS) qui a apporté le premier financement de cette étude ainsi que le Programme d'Appui Stratégique à la Recherche Scientifique (PASRES). Nous sommes grandement redevables à tous les acteurs à tous les niveaux de la chaîne de valeur de bambou, y compris les agriculteurs et toutes les populations de AbbèBéguini, Azaguié-Ahoua, Azaguié-Gare et M'Bromé pour leur coopération sans réserve et la fourniture de données pendant le temps de l'enquête. Nos sincères remerciements vont aussi aux deux relecteurs anonymes pour leur précieuse contribution améliorer la qualité d'une version antérieure du manuscrit.

\section{Références citées}

Akaike H. 1973. La théorie de l'information comme une extension de principe du maximum de vraisemblance. Petrov B. N., Csaki F. (eds). Deuxième symposium international sur la théorie de l'information. Budapest, Hongrie, 142p. 
Assogba GA, Fandohan AB, Salako VK, Assogbadjo AE. 2017. Usages de Bombax costatum (Malvaceae) dans les terroirs riverains de la réserve de biosphère de la Pendjari, République du Bénin. Bois et Forêts des Tropiques 333(3):17-29.

Assogbadjo AE, Glèlè Kakaï $R$, Chadaré $F J$, Thomson L, Kyndt T, Sinsin B, Van Damme P. 2008. Classement folklorique, la perception et les préférences des produits du baobab en Occident Afrique: Conséquences pour la conservation et l'amélioration des espèces. Botanique économique 62:74-84.

Babulo B, Muys B, Nega F, Tollens E, Nyssen J, Deckers J, Mathijs E. 2009. The economic contribution of forest resource use to rural livelihoods in Tigray, Northern Ethiopia. Forest Policy Economic 11:109-117.

Belem B, 2009. Ethnobotanique et conservation de Bombax costatum Pel. \& Vuil. (faux kapokier) dans les systèmes de production agricoles du plateau central, Burkina Faso. Thèse de doctorat, Université de Ouagadougou, Burkina Faso, 189 p.

Belem B, Smith OC, Theilade I, Bellefontaine R, Guinko S, Lykke AM. 2008. Identification des arbres hors forêts préférées des populations du Sanmatenga (Burkina Faso). Bois et forêts des tropiques 298(4):53-64.

Bitariho R, McNeilage A. 2008. Population structure of montane bamboo and causes of its decline in Echuya Central Forest Reserve, South West Uganda. African Journal of Ecology 46:325-332.

Bussmann RW, Paniagua-Zambrana NY, Kuhlman A, Rakotoarivony F, Razanatsima A, Rakotoarivelo N, Razafitsalama JL, Randrianasolo A. 2015. Palms and palm uses in Ambalabe, a community in Eastern Madagascar. Ethnobotany Research \& Applications 14:17-26.

Camou-Guerrero A, Reyes-Garcia, Martinez-Ramos M, Cassa A. 2008. Knowledge and use value of plant species in a Raramuri community: a gender perspective for conservation. Human Ecology 36:259-272.

Cavendish W. 2000. Empirical regularities in the rural environment: evidence from Zimbabwe. World Development 28(11):1979-2003.

Creswell JW. 2003. Research design: Qualitative, quantitative, and mixed method approaches. Thousand Oaks, California et al: SAGE Publications.

Darko Obiri B, Oteng-Amoako AA. 2007. Towards a sustainable development of the bamboo industry in Ghana. Ghana Journal of Forestry 21\&22:14-27.

Desalegn G, Tadesse W. 2014. Resource Communication. Resource potential of bamboo, challenges and future directions towards sustainable management and utilization in Ethiopia. Forest System 23(2):294-299.
Dje Bi DPV, Koffi JK, Vroh BTA, Kpangui KB, Adou Yao CY. 2017. Exploitation et importance socioéconomique du bambou de chine, Bambusa vulgaris Schrad. ex J.C. Wendl. (Poaceae) dans la région de l'Agnéby-Tiassa : cas de la Sous-Préfecture d'Azaguié (Sud-Est de la Côte d'Ivoire). International Journal Biological and Chemical Science 11(6):2887-2900.

Dransfield S, Widjaja EA. 1995. Bambous : Plantes ressources d'Asie Du Sud-Est No. 7 Leiden: Éditeurs Backhuys. $123 \mathrm{p}$.

Dytham C. 2011. Choosing and using statistics: a biologist's guide. 3rd ed. Chichester: Blackwell Science.

Fandohan B, Assogbadjo AE, Glèlè KRL, Sinsin B. 2010. Effectiveness of a protected areas network in the conservation of Tamarindus indica (Leguminosea Caesalpinioideae) in Benin. African Journal of Ecology 49:40-50.

Fandohan AB, Gouwakinnou NG, Koko KIE, Deléké $M$, Glèlè KR, Assogbadjo AE. 2015. Domesticating and conserving indigenous trees species: an ecosystem based approach for adaptation to climate change in Sub-Sahara Africa. CAMES (African and Malagasy Council for Higher Education). Life Sciences, Earth Sciences and Agronomy 3(1):55-60.

FAO. 2001. Resource Assessment of Non-Wood Forest Products, Experience and biometric principles. Non-Wood Forest Products 13. FAO Rome, 139p.

FAO. 2007. World bamboo resources: a thematic study prepared in the framework of the Global Forest Resources Assessment 2005, Rome, 149p.

Fayera B, Tsegaye B, Teshale W. 2016. Value chain analysis of lowland bamboo products: The case of Homosha district, Benishangul Gumuz Regional State. African Journal of Marketing Management 6(10):1-15.

Gnahoua GM, Nguessan KA, Ballé P. 2014. Les jachères de légumineuses arborescentes: sources potentielles de bois énergie et de service en Côte d'Ivoire. Journal Applied Biosciences 73:7290-7297.

Gomez-Beloz A. 2002. La connaissance de l'utilisation des plantes de la Winikina Warao: Le cas des questionnaires en ethnobotanique. Economique Botany 56:231-241.

Gouwakinnou GN, Lykke AM, Assogbadjo AE, Sinsin B. 2011. Local knowledge, pattern and diversity of use of Sclerocarya birrea. Journal of Ethnobiology and Ethnomedicine 7:8-15.

Gruca M, Blach-Overgaard A, Balslev H. 2015. Afrique ethno-médecine du palmier. Journal d'ethnopharmacologie 165: 227-237.

Honfo H, Tovissodé FC, Gnanglè C, Mensah S, Salako VK, Assogbadjo AE. 2015. Connaissances 
traditionnelles et valeur du bambou dans le sud-est du Bénin: implications pour gestion durable. Ethnobotanique Recherche et Applications 14:139153.

Houehanou TD, Assogbadjo A, Kakaï RG, Houinato M, Sinsin B. 2011. Évaluation des utilisations préférées locales et traditionnelles connaissances écologiques en relation avec trois usages multiples espèces d'arbres au Bénin (Afrique de l'Ouest). Politique forestière et Économie 13 (7):554-562.

Ingram V, Bongers G. 2009. Valuation of NonTimber Forest Product Chains in Congo Basin: A methodology for valuation. CIFOR, Younde, Cameroon, FAO-CIFOR-SNV-World Agroforestry Center-COMIFAC, $80 \mathrm{p}$.

Johnson JB, Omland KS. 2004. Sélection du modèle en écologie et l'évolution. Tendances en matière d'écologie et d'évolution 19(2):101-108.

Karanja PN, Kenji GM, Njoroge SM, Sila DN, Onyango CA, Koaze H, Baba N. 2015. Variation des nutriments et des propriétés fonctionnelles chez les jeunes pousses d'une espèce de bambou (Yushania alpina) en croissance à Mt. Région d'Elgon dans l'ouest du Kenya. Journal of Food and Nutrition Research 3(10):675-680.

Kleinhenz V, Midmore DJ. 2001. Aspects of bamboo agronomy. Advances in Agronomy 74:99-153.

Koulibaly A, Monian M, Ackah JAAB, Kone MWTK. 2016. Étude ethnobotanique des plantes médicinales: cas des affections les plus fréquentes d'une région agricole Daloa (Centre Ouest, Côte d'Ivoire). Journal of Animal \& Plant Sciences 31(2):5021-5032

Larpkern P, Moe SR, Totland Ø. 2011. Bamboo dominance reduces tree regeneration in disturbed rainforest. Oecologia 165:161-168.

Lescuyer G. 2010. Importance économique des produits forestiers non ligneux dans quelques villages du Sud-Cameroun. Bois et forêts des tropiques 304 (2):15-24.

Louppe D, Oteng-Amoako AA, Bord M. 2008. Bois de construction. PROTA 1:100-103.

Mahapatra AK, Albers HJ, Robinson EJZ. 2005. L'Impact des ventes de PFNL sur le revenu monétaire des ménages ruraux La forêt sèche décidue de I'Inde. Gestion de l'environnement 35 (3):258-265.

Mekonnen Z, Worku A, Yohannes T, Alebachew M, Teketay D, Kassa H. 2014. Bamboo Resources in Ethiopia: Value chain and contribution to livelihoods. Ethnobotanical Ressources Applied 12:511-524.

Mertens B, Hua L, Belcher B, Ruiz-Pérez M, Fu M, Yang X. 2008. Modèles spatiaux et processus d'expansion du bambou en Chine Du sud. Géographie appliquée 28 (1): 16-31.
Nath AJ, Franklin DC, Lawes MJ, Das MC, Das AK. 2011. Impact of culm harvest on seed production in a Monocarpic Bamboo. Biotropica 44:699-704.

N'Guessan K. 2008. Plantes médicinales et pratiques médicales traditionnelles chez les peuples Abbey et Krobou du département d'Agboville (Côte d'Ivoire). Thèse de Doctorat d'Etat ès Sciences, Université de Cocody-Abidjan. 235 p.

Morris J, Bay A. 2002. An overview of the NTFP subsector in Vietnam. Forest science institute of Vietnam, NTFP Research Centre: Vietnam.

Mukul SA, Parvez-Rana M. 2013. The trade of bamboo (Graminae) and its secondary products in a regional market of southern Bangladesh: status and socio-economic significance. International Journal of Biodiversity Science, Ecosystem Services \& Management 9(2):146-154.

Nirmala C, Bisht MS, Sharma V. 2014. Bioactive compounds in bamboo shoots: Health benefits and prospects for developing nutraceuticals. In Sharma GJ, Sharma RN eds. Current Topics in Redox Biology pp. 82-100, MC Graw Hill, New Delhi, India.

Ogunjinmi AA, ljeomah HM, Aiyeloja AA. 2009. Socio-economic importance of bamboo (Bambusa vulgaris) in Borgu local government area of Niger State, Nigeria. Journal of Sustainable Development in Africa 10(4):284-298.

Ohrnberger D. 1999. Les bambous du monde : Nomenclature annotée et littérature de l'espèce et le plus haut et inférieur. Brochures de la biologie 741:279-292.

Perez RM, Byron N. 1999. A Methodology to analyze divergent case studies of Non-Timber Forest Products and their development potential. Forest Science 45(1):1-14.

Puri HS. 2003. Rasayana ayurvedic herbs for longevity and rejuvenation. Taylor and Francis, London, 71-73.

Rao AN, Rao VR. 1998. Bamboo-conservation, diversity, ecogeography, germplasm, resource utilization and taxonomy. Proceedings of training course cum Workshop, Kunming and Xishuanbanna, Yunnan, China.

R Core Team. 2016. A: A language and environment for statistical computing. Vienna, Austria, R Foundation for Statistical Computing.

Rashford J. 2015. The uses of the baobab flower (Adansonia digitata L.). Ethnobotany Research and Applications 14:211-229

RGPH. 2014. Recensement Général de la Population et de l'Habitat (RGPH) 2014.

Rui-Perez PJ, Alarcon ZB, Mendoza MGD, Barcena GR, Hernandez GA, Herrera HJG. 2001. Response of kudzu as protein bank on steers grazing African 
stargrass. Technology Pectoral of Mexico 39(1):3952.

Salam K. 2008. India, CBTC, Guwahati, Bamboo for economic prosperity and ecological security. www.indianfolklore.org/journals/index.php/ishani/arti cle/viewPDFInterstitial/409/353.

Shackleton C, Shackleton S. 2004. The importance of non-timber forest products in rural livelihood security and as safety nets: A review of evidence from South Africa. South African Journal of Science 100:658-664.

Sofowara A. 2010. Plantes médicinales et médecine traditionnelle d'Afrique. Paris, France, Karthala, 378 p.

Tardio J, Pardo-De-Santayana M. 2008. Importance culturelle indices: une analyse comparative basée sur le sauvage utile plantes du sud de la Cantabrie (nord de l'Espagne). Economique Botanique 62(1):24-39.

Tefera B, Lindner A, Pretzsch J. 2013. Indicators and determinants of Small-Scale Bamboo commercialization in Ethiopia. Forests 4(3):710-729. Tesfaye Y, Roos A, Campbell B, Bohlin F. 2011. Forest incomes and poverty alleviation under participatory forest management in the Bale Highlands, Southern Ethiopia. International Forestry Review 12(1):66-77.

Vedeld P, Angelsen A, Bojo J, Sjaastad E, Kobugabe BG. 2007. Forest environmental incomes and the rural poor. Forest Policy Economic 9:869-879.

Vroh BTA, Adou Yao CY, Kouamé D, N'Da DH, N'Guessan KE. 2010. Diversité floristique et structurale sur le site d'une réserve naturelle volontaire à Azaguié, Sud-Est de la Côte d'Ivoire. European Journal of Scientific Research 63(3):40114021.

Yakubu M T, Bukoye BB. 2005. Les possibilités Abortif" de l'extrait aqueux de Bambusa vulgaris sur les lapines hollandaises enceintes", Pub Med, Centre National pour l'Information de la Biotechnologie, Etats-Unis Bibliothèque Nationale de Médecine. Pp 62-74.

Zadou DA, Koné I, Mouroufié VK, Adou Yao CY, Gléanou KE, Kablan YA, Coulibaly D, Ibo JG. 2011. Valeur de la Forêt des Marais Tanoe-Ehy (sud-est de la COTE D'IVOIRE) pour la conservation: dimension socio-anthropologique. Tropical Conservation Science 4(4):373-385. 